\title{
STABILITY OF TRIVIAL TORUS OF NONLINEAR MULTIFREQUENCY SYSTEMS VIA SIGN-DEFINITE QUADRATIC FORMS
}

\author{
PETRO FEKETA, OLENA A. KAPUSTIAN, AND MYKOLA M. PERESTYUK \\ Received 28 February, 2018
}

\begin{abstract}
This paper studies stability properties of the trivial invariant torus of a class of nonlinear extensions of dynamical systems on torus. Two theorems on exponential stability and instability of the invariant torus in terms of quadratic forms that are sign-definite in nonwandering set of the dynamical system have been proven.
\end{abstract}

2010 Mathematics Subject Classification: 34C46; 34C45; 34D35

Keywords: invariant tori, multifrequency oscillations, stability

\section{INTRODUCTION}

Fundamental results of the qualitative theory of multifrequency oscillations, in particular, the problems of the existence and stability of invariant sets of dynamical systems defined in the direct product of $m$-dimensional torus and $n$-dimensional Euclidean space, have been developed by A. M. Samoilenko and summarized in [12]. In [6], the stability properties of invariant tori have been studied in terms of signdefinite quadratic forms. In this paper, we establish new conditions for exponential stability and instability of the trivial invariant torus of nonlinear extension of dynamical system on torus which are formulated in terms of quadratic forms that are sign-definite not on the entire surface of the torus, but in nonwandering set [7] of dynamical system on torus only. The corresponding results for linear extensions of dynamical systems on torus have been obtained in $[1,3,8-11]$.

\section{MotivATION AND PROBLEM STATEMENT}

We consider a system of ordinary differential equations defined in the direct product of $m$-dimensional torus $\mathcal{T}_{m}$ and $n$-dimensional Euclidean space $\mathbb{R}^{n}$

$$
\frac{d \varphi}{d t}=a(\varphi), \quad \frac{d x}{d t}=P(\varphi, x) x,
$$


where $\varphi=\left(\varphi_{1}, \ldots, \varphi_{m}\right)^{T} \in \mathcal{T}_{m}, x=\left(x_{1}, \ldots, x_{n}\right)^{T} \in \mathbb{R}^{n}$, function $P$ is continuous in $\mathcal{T}_{m} \times \mathbb{R}^{n}$ and for every $x \in \mathbb{R}^{n} P(\cdot, x), a(\cdot) \in C\left(\mathcal{T}_{m}\right) ; C\left(\mathcal{T}_{m}\right)$ is a space of continuous $2 \pi$-periodic with respect to each of the component $\varphi_{v}, v=1, \ldots, m$ functions defined on $\mathcal{T}_{m}$.

We assume that the following conditions hold:

$$
\begin{gathered}
\exists M>0 \text { such that } \forall(\varphi, x) \in \mathcal{T}_{m} \times \mathbb{R}^{n}\|P(\varphi, x)\| \leq M ; \\
\forall r>0 \exists L=L(r)>0 \text { such that } \forall x^{\prime}, x^{\prime \prime},\left\|x^{\prime}\right\| \leq r,\left\|x^{\prime \prime}\right\| \leq r, \forall \varphi \in \mathcal{T}_{m} \\
\left\|P\left(\varphi, x^{\prime \prime}\right)-P\left(\varphi, x^{\prime}\right)\right\| \leq L\left\|x^{\prime \prime}-x^{\prime}\right\| ; \\
\exists A>0 \forall \varphi^{\prime}, \varphi^{\prime \prime} \in \mathcal{T}_{m}\left\|a\left(\varphi^{\prime \prime}\right)-a\left(\varphi^{\prime}\right)\right\| \leq A\left\|\varphi^{\prime \prime}-\varphi^{\prime}\right\| .
\end{gathered}
$$

Condition (2.4) guarantees that the system

$$
\frac{d \varphi}{d t}=a(\varphi)
$$

generates a dynamical system on $\mathcal{T}_{m}$, which we will denote by $\varphi_{t}(\varphi)$.

Definition 1 ([7]). A point $\varphi \in \mathcal{T}_{m}$ is called a nonwandering point of dynamical system (2.5) if there exist a neighbourhood $U(\varphi)$ and a moment of time $T=T(\varphi)>0$ such that

$$
U(\varphi) \cap \varphi_{t}(U(\varphi))=\varnothing \quad \forall t \geq T .
$$

Let us denote by $\Omega$ a set of all nonwandering points of dynamical system (2.5). Since $\mathcal{T}_{m}$ is a compact set, the set $\Omega$ is nonempty, invariant, and compact subset of $\mathcal{T}_{m}$ [12]. Additionally, the following holds:

Lemma 1 ([7]). For any $\varepsilon>0$ there exist $T(\varepsilon)>0$ and $N(\varepsilon)>0$ such that for any $\varphi \notin \Omega$ the corresponding trajectory $\varphi_{t}(\varphi)$ spends only a finite time that is bounded by $T(\varepsilon)$ outside the $\varepsilon$-neighbourhood of the set $\Omega$, and leaves this set not more than $N(\varepsilon)$ times.

Definition 2 ([12]). Trivial invariant torus

$$
x=0, \quad \varphi \in \mathcal{T}_{m}
$$

of the system (2.1) is called exponentially stable if there exist constants $K>0, \gamma>0$, and $\delta>0$ such that for all $\varphi \in \mathcal{T}_{m}$ and for all $x^{0} \in \mathbb{R}^{n},\left\|x^{0}\right\| \leq \delta$ it holds that

$$
\forall t \geq 0 \quad\left\|x\left(t, \varphi, x^{0}\right)\right\| \leq K\left\|x^{0}\right\| e^{-\gamma t},
$$

where $x\left(t, \varphi, x^{0}\right)$ is a solution to the Cauchy problem

$$
\frac{d x}{d t}=P\left(\varphi_{t}(\varphi), x\right) x, \quad x(0)=x^{0} .
$$

In [5], the conditions for the exponential stability of the trivial invariant torus of the system (2.1) have been established in terms of the properties of function $\varphi \mapsto P(\varphi, 0)$ in the nonwandering set $\Omega$ of dynamical system (2.5): 
Lemma 2 ([5]). Let

$$
\forall \varphi \in \Omega \quad \lambda(\varphi, 0)<0,
$$

where $\lambda(\varphi, x)$ is the largest eigenvalue of the matrix

$$
\hat{P}(\varphi, x)=\frac{1}{2}\left(P(\varphi, x)+P^{T}(\varphi, x)\right) .
$$

Then, the trivial invariant torus of system (2.1) is exponentially stable.

The following example demonstrates the case when the trivial invariant torus is exponentially stable (this will be proven in Theorem 1), however the condition (2.8) does not hold.

Example 1. Consider a system defined in $\widetilde{T}_{1} \times \mathbb{R}^{2}$

$$
\begin{gathered}
\frac{d \varphi}{d t}=-\sin ^{2}\left(\frac{\varphi}{2}\right) \\
\left(\begin{array}{cc}
\frac{d x_{1}}{d t} \\
\frac{d x_{2}}{d t}
\end{array}\right)=\left(\begin{array}{cc}
\sin \left(\varphi+x_{1}+x_{2}\right) x_{1} & -x_{2} \\
x_{1} & -\sin \left(x_{1}-x_{2}-\varphi\right) x_{2}
\end{array}\right) .
\end{gathered}
$$

Dynamical system on torus $\mathcal{T}_{1}$ that are generated by (2.9) has a nonwandering set

$$
\Omega=\{\varphi=0\} .
$$

However, the matrix

does not fulfill condition (2.8).

$$
\hat{P}(0, \overline{0})=\left(\begin{array}{ll}
0 & 0 \\
0 & 0
\end{array}\right)
$$

The main results of this paper are theorems on exponential stability and instability of the trivial torus of the systems of a class (2.9) with conditions given in terms of quadratic forms that are sign-definite in nonwandering set $\Omega$ of dynamical system on torus only.

\section{MAIN RESULTS}

For any $\varphi \in \mathcal{T}_{m}, x \in \mathbb{R}^{n}$ let us denote

$$
\begin{aligned}
\hat{S}(\varphi, x) & =\frac{\partial S(\varphi, x)}{\partial \varphi} a(\varphi)+\frac{\partial S(\varphi, x)}{\partial x}(P(\varphi, x) x) \\
& +S(\varphi, x) P(\varphi, x)+P^{T}(\varphi, x) S(\varphi, x),
\end{aligned}
$$

where $S=S(\varphi, x)$ is a symmetric matrix of a class $C^{1}\left(\mathcal{T}_{m} \times \mathbb{R}^{n}\right)$.

Theorem 1. Let there exist a symmetric matrix $S=S(\varphi, x)$ of the class $C^{1}\left(\mathcal{T}_{m} \times\right.$ $\mathbb{R}^{n}$ ) such that

$$
\forall \varphi \in \Omega \quad S(\varphi, 0)>0, \quad \hat{S}(\varphi, 0)<0 .
$$

Then, the trivial torus of system (2.1) is exponentially stable. 
Proof. Due to the conditions (3.2) and continuous dependence of the polynomialFLs roots on the coefficients [4], we get that for some $r>0, \gamma>0$ the following inequalities hold

$$
\forall \varphi \in O_{r}(\Omega) \forall x \in \mathbb{R}^{n},\|x\|<r \quad S(\varphi, x) \geq \gamma E, \quad \hat{S}(\varphi, x) \leq-\gamma E .
$$

Also, there exists a constant $C=C(r)>0$ such that for all $\varphi \in T_{m}$ and for all $x \in \mathbb{R}^{n}$, $\|x\| \leq r$

$$
\|S(\varphi, x)\|+\|\hat{S}(\varphi, x)\| \leq C
$$

Denote by

$$
V(\varphi, x)=(S(\varphi, x) x, x)
$$

Let

$$
\varphi \in O_{r}(\Omega) \quad \text { and } \quad \forall s \geq 0 \quad \varphi_{s}(\varphi) \in O_{r}(\Omega) .
$$

Then, for the solution to (2.7) $x(t)=x\left(t, \varphi, x^{0}\right)$ with $\left\|x^{0}\right\|<r$ the following estimate $\|x(t)\|<r$ holds for $t \in[0, T), 0<T \leq+\infty$. Hence, from (3.3),

$$
\begin{gathered}
\gamma\|x(t)\|^{2} \leq V\left(\varphi_{t}(\varphi), x(t)\right) \leq C\|x(t)\|^{2}, \\
\frac{d}{d t} V\left(\varphi_{t}(\varphi), x(t)\right) \leq-\gamma\|x(t)\|^{2}
\end{gathered}
$$

hold for $t \in[0, T)$. From the last inequalities we get that

$$
V\left(\varphi_{t}(\varphi), x(t)\right) \leq V\left(\varphi, x^{0}\right) e^{-\frac{\gamma}{C} t} .
$$

Hence, there exist constants $K_{1}>0$ and $\gamma_{1}>0$ such that $\forall t \in[0, T)$

$$
\|x(t)\| \leq K_{1}\left\|x^{0}\right\| e^{-\gamma_{1} t} .
$$

For $\left\|x^{0}\right\|<\frac{r}{K_{1}}$ we obtain that $T=+\infty$ and the inequality (3.6) holds for all $t \geq 0$.

Now, let $\varphi \in O_{r}(\Omega)$, but there exists $t_{1}>0$ such that

$$
\forall t \in\left[0, t_{1}\right) \quad \varphi_{t}(\varphi) \in O_{r}(\Omega), \varphi_{t_{1}}(\varphi) \notin O_{r}(\Omega) .
$$

From Lemma 1, there exist

$$
\begin{gathered}
N(\varphi, r) \leq N(r), \quad\left\{\tau_{i}(\varphi, r)\right\}_{i=1}^{N(\varphi, r)+1}, \quad\left\{t_{i}(\varphi, r)\right\}_{i=1}^{N(\varphi, r)} \\
\sum_{i=1}^{N(\varphi, r)+1} \tau_{i}(\varphi, r)=: T(\varphi, r) \leq T(r)
\end{gathered}
$$

such that

$$
\begin{gathered}
\varphi_{t}(\varphi) \in O_{r}(\Omega) \\
\forall t \in\left(0, t_{1}\right) \cup \bigcup_{k=1}^{N(\varphi, r)-1}\left(\sum_{i=1}^{k}\left(\tau_{i}+t_{i}\right), \sum_{i=1}^{k}\left(\tau_{i}+t_{i+1}\right)\right) \bigcup\left(\sum_{i=1}^{N(\varphi, r)}\left(\tau_{i}+t_{i}\right),+\infty\right) .
\end{gathered}
$$


Then, for $t \in\left[0, t_{1}\right]$

$$
\|x(t)\| \leq K_{1}\left\|x^{0}\right\| e^{-\gamma_{1} t}<r \quad \text { if } \quad\left\|x^{0}\right\|<\frac{r}{K_{1}} .
$$

For $t \in\left[t_{1}, t_{1}+\tau_{1}\right]$, from (2.2) and Wazewski inequality [2],

$$
\begin{aligned}
\|x(t)\| & \leq\left\|x\left(t_{1}\right)\right\| e^{M\left(t-t_{1}\right)} \\
& \leq K_{1}\left\|x^{0}\right\| e^{\left(\gamma_{1}+M\right) \tau_{1}} e^{-\gamma_{1} t} \\
& <r \quad \text { if }\left\|x^{0}\right\|<\frac{r}{K_{1} e^{\left(\gamma_{1}+M\right) \tau_{1}} .} .
\end{aligned}
$$

For $t \in\left[t_{1}+\tau_{1}, t_{1}+\tau_{1}+t_{2}\right]$,

$$
\|x(t)\| \leq K_{1}^{2}\left\|x^{0}\right\| e^{\left(\gamma_{1}+M\right) \tau_{1}} e^{-\gamma_{1} t}<r \quad \text { if } \quad\left\|x^{0}\right\|<\frac{r}{K_{1}^{2} e^{\left(\gamma_{1}+M\right) \tau_{1}}} .
$$

Continuing this process, due to (3.7), finally we get:

$$
\text { for } \begin{gathered}
K:=K_{1}^{N(r)} e^{(\gamma+M) T(r)}, \quad \delta:=\frac{r}{K_{1}^{N(r)} e^{(\gamma+M) T(r)}}, \quad\left\|x^{0}\right\|<\delta, \\
\forall t \geq 0 \quad\|x(t)\| \leq K\left\|x^{0}\right\| e^{-\gamma_{1} t} .
\end{gathered}
$$

Now, let us consider the case of $\varphi \notin O_{r}(\Omega)$. In this case,

$$
\forall \tau_{0} \in(0, T(r)) \quad \varphi_{\tau_{0}}(\varphi) \in O_{r}(\Omega)
$$

and

$$
\forall t \in\left[0, \tau_{0}\right] \quad\|x(t)\| \leq\left\|x^{0}\right\| e^{\left(M+\gamma_{1}\right) T(r)} \| e^{-\gamma_{1} t} .
$$

Then, for

$$
\hat{K}=K e^{(\gamma+M) T(r)}, \quad \hat{\delta}=\frac{\delta}{e^{(\gamma+M) T(r)}}
$$

we obtain the required estimate (2.6). This completes the proof.

Example 2 (revisited). Let us illustrate the usage of Theorem 1 for the system (2.9), (2.10). Let

$$
S=S(\varphi, x)=\left(\begin{array}{ll}
2 & 1 \\
1 & 1
\end{array}\right)>0
$$

Then,

$$
\hat{S}(0, \overline{0})=\left(\begin{array}{cc}
2 & -1 \\
-1 & -2
\end{array}\right)<0
$$

which guarantee exponential stability of the trivial invariant torus.

The following theorem provides sufficient conditions for instability of the trivial torus of system (2.1) in terms of sign-definite on the set $\Omega$ quadratic forms. 
Theorem 2. Let there exist a symmetric matrix $S=S(\varphi, x)$ of the class $C^{1}\left(\mathcal{T}_{m} \times\right.$ $\mathbb{R}^{n}$ ) such that for the matrix (3.1) and for the quadratic form (3.5) the following conditions hold:

$$
\forall \varphi \in \Omega \quad \hat{S}(\varphi, 0)>0,
$$

$\forall \delta>0 \exists x_{0} \in \mathbb{R}^{n},\left\|x_{0}\right\|<\delta, \exists \varphi_{0} \in \Omega \quad$ such that $V\left(\varphi_{0}, x_{0}\right)>0$.

Then, the trivial torus of system (2.1) is unstable.

Proof. From (3.9) and (3.3), $\exists \beta>0$ and $\exists r>0$ such that

$$
\forall \varphi \in \Omega \quad \forall x \in \mathbb{R}^{n},\|x\|<r \quad \hat{S}(\varphi, x) \geq \beta E .
$$

Let for arbitrary $\delta>0(\delta<r)$, the corresponding $x_{0}, \varphi_{0}$ are from the condition (3.10), and $x(t)=x\left(t, \varphi_{0}, x_{0}\right)$ is the solution to the Cauchy problem (2.7). Next, we show that $\exists t_{1}>0$ such that $\left\|x\left(t_{1}\right)\right\|=r$. This will be sufficient to prove instability.

Suppose the opposite: Let

$$
\forall t \geq 0 \quad\|x(t)\|<r .
$$

Then, for the function $v(t)=V\left(\varphi_{t}\left(\varphi_{0}\right), x(t)\right)$ we have:

$$
\frac{d}{d t} v(t) \geq \beta\|x(t)\|^{2} .
$$

From the condition (3.10), $v(0)=V\left(\varphi_{0}, x_{0}\right)=\alpha>0$. Hence,

$$
\forall t \geq 0 \quad v(t) \geq \alpha .
$$

Then, $\exists \varepsilon>0$ such that $\forall t \geq 0\|x(t)\| \geq \varepsilon$. Really, if it is not so then there exists a sequence $t_{k} \rightarrow \infty$ such that $\left\|x\left(t_{k}\right)\right\| \rightarrow 0$. Finally,

$$
v\left(t_{k}\right)=\left(S\left(\varphi_{t_{k}}\left(\varphi_{0}\right), x\left(t_{k}\right)\right) x\left(t_{k}\right), x\left(t_{k}\right)\right) \leq C\left\|x\left(t_{k}\right)\right\|^{2} \rightarrow 0,
$$

where constant $C>0$ is from (3.4), which contradicts (3.12).

Then, from (3.11):

$$
v(t) \geq \alpha+\varepsilon^{2} t \quad \forall t \geq 0 .
$$

Hence, $\forall t \geq 0$

$$
\|x(t)\|^{2} \geq \frac{1}{C}\left(\alpha+\varepsilon^{2} t\right) .
$$

The latter estimate implies the existence of $t_{1}>0$ such that $\left\|x\left(t_{1}\right)\right\|=r$. This completes the proof. 


\section{REFERENCES}

[1] F. Asrorov, Y. Perestyuk, and P. Feketa, "On the stability of invariant tori of a class of dynamical systems with the Lappo-Danilevskii condition," Memoirs on Differential Equations and Mathematical Physics, vol. 72, pp. 15-25, 2017.

[2] B. Demidovich, "Lektsii po matematicheskoi teorii ustoichivosti." Moskau: Verlag 'Nauka'. Hauptredaktion für physikalisch-mathematische Literatur. 472 S. (1967)., 1967.

[3] P. Feketa and Y. Perestyuk, "Perturbation theorems for a multifrequency system with pulses." $J$. Math. Sci., New York, vol. 217, no. 4, pp. 515-524, 2016, doi: 10.1007/s10958-016-2988-6.

[4] R. A. Horn and C. R. Johnson, Matrix analysis., 2nd ed. Cambridge: Cambridge University Press, 2013.

[5] O. Kapustyan, F. Asrorov, and Y. Perestyuk, "On exponential stability of the trivial torus for a certain class of nonlinear impulsive systems," Nonlinear Oscil., vol. 20, no. 4, pp. 502-508, 2017.

[6] Y. A. Mitropolsky, A. M. Samoilenko, and V. L. Kulik, Dichotomies and stability in nonautonomous linear systems. CRC Press, 2002.

[7] V. Nemytskii and V. Stepanov, "Qualitative theory of differential equations." (Princeton Mathematical Series). New Jersey: Princeton University Press. VIII, 523 p. (1960)., 1960.

[8] M. M. Perestyuk and Y. M. Perestyuk, "On the stability of toroidal manifold for one class of dynamical systems," Journal of Mathematical Sciences, vol. 228, no. 3, pp. 314-322, 2018, doi: 10.1007/s10958-017-3623-x.

[9] M. Perestyuk and P. Feketa, "Invariant manifolds of one class of systems of impulsive differential equations." Nonlinear Oscil., N.Y., vol. 13, no. 2, pp. 260-273, 2010, doi: 10.1007/s11072-0100112-2.

[10] M. Perestyuk and P. Feketa, "On preservation of the invariant torus for multifrequency systems." Ukr. Math. J., vol. 65, no. 11, pp. 1661-1669, 2014, doi: 10.1007/s11253-014-0887-x.

[11] M. Perestyuk and P. Feketa, "Invariant sets of impulsive differential equations with particularities in $\omega$-limit set." Abstr. Appl. Anal., vol. 2011, p. 14, 2011, doi: 10.1155/2011/970469.

[12] A. Samoilenko, Elements of the mathematical theory of multi-frequency oscillations. Dordrecht etc.: Kluwer Academic Publishers, 1991.

Authors' addresses

\section{Petro Feketa}

University of Kaiserslautern, Department of Mechanical and Process Engineering, Gottlieb-DaimlerStraße, Postfach 3049, 67663 Kaiserslautern, Germany

E-mail address: petro.feketa@mv.uni-kl.de

Olena A. Kapustian

Taras Shevchenko National University of Kyiv, Faculty of Computer Science and Cybernetics,

Volodymyrska Street 64, 01601 Kyiv, Ukraine

E-mail address: olena.kap@gmail.com

Mykola M. Perestyuk

Taras Shevchenko National University of Kyiv, Faculty of Mechanics and Mathematics, Volodymyrska Street 64, 01601 Kyiv, Ukraine

E-mail address: perestyuknnegmail.com 